\title{
FROM THE CONVEX GEOMETRY OF CERTAIN VALUATIONS TO POSITIVITY ASPECTS IN ALGEBRAIC GEOMETRY
}

\author{
VICTOR LOZOVANU
}

One of the earliest known theorems in the history of mathematics is the Pythagorean theorem. Its proof, due to Pythagora (c. 570-c. 495 BC), can be explained by the following two pictures:

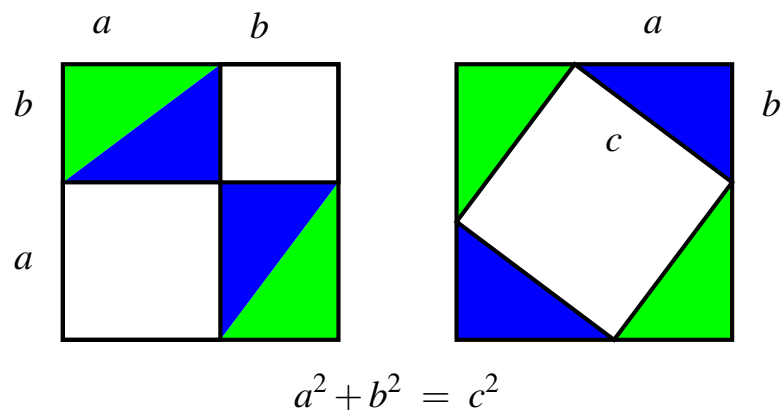

Comparing the area of the "white" region in the first picture with the area of the "white" region in the second implies the Pythagorean identity. Furthermore, it's captivating how the proof uses convex geometry (Euclidean geometry of polygons) to explore algebraic equations.

This philosophy is revived much later in a spectacular but simple fashion by the work of Newton. In a letter to Oldenburg from 1671, Newton has the idea to associate to a polynomial in two variables $f=\sum_{i, j} a_{i j} x^{i} y^{j} \in \mathbb{C}[x, y]$ a convex set, called the Newton polygon, as follows

$$
\mathbb{R}^{2} \supseteq \Delta(f) \stackrel{\text { def }}{=} \text { convex hull }\left\{(i, j) \in \mathbb{Z}^{2} \mid a_{i j} \neq 0\right\},
$$

extending the idea of the degree of a polynomial to a polygon. Using the shape of $\Delta(f)$, Newton describes then an exhaustive algorithm that finds all the solutions $y=y(x)$ of the equation $f(x, y)=0$ as Puiseux series. It is worth noting here that if one thinks of the equation $f(x, y)=0$ as an affine curve $C \subseteq \mathbb{C}^{2}$, then this is equivalent to constructing a resolution of singularities of the curve $C$ (see Chapter I in [Ko07] for a nice exposition of these ideas).

After the work of Newton, the above convex construction has slowly found many applications in mathematics. In algebraic geometry it was pioneered by Arnold, who conjectured that it might be possible to express many invariants associated to a holomorphic function in terms of its Newton polygon, at least for "almost all functions" with a given polygon. This has been worked out in the 1970s by Arnold's school (D. Bernstein, A. Khovanskii, A. Kouschnirenko and A. Varchenko).

The idea, originally due to Khovanskii, is to use the polygon $\Delta:=\Delta(f)$ to construct a compactification $X(\Delta)$ of $\mathbb{C}^{2}$ by adding projective lines at infinity. The projective surface $X(\Delta)$ is special and is called a toric surface. Toric varieties in general, originally introduced by Demazure in 1970, are a very special class of projective varieties constructed from convex geometric data (polytopes, cones, fans etc.). Thus many properties/invariants of the toric variety can be translated into the convex geometric world and viceversa. In our case, then studying $f \in \mathbb{C}[x, y]$ is equivalent to doing the same for the divisor $\bar{C} \subseteq X(\Delta)$, the compactification 
of $C:=\{f=0\}$. Consequently, using the toric language, to study the geometry of $\bar{C}$ is the same as to explore the Euclidean geometry of $\Delta(f)$.

It's worth mentioning here two results that captures nicely not only how can convex geometry be used to study algebraic varieties, but also how to explore convex shapes using algebraic geometry.

Theorem. (1) (Khovanskii) Let $C \subseteq \mathbb{C}^{2}$ be an irreducible curve defined by a polynomial $f \in \mathbb{C}[x, y]$. Then the topological genus $g(C)$ is at most the number of integral points contained in the interior of the Newton polygon $\Delta(f)$. Equality happens when $f$ is chosen to be generic with a fixed Newton polygon.

(2) (Bernstein) If $\Delta \subseteq \mathbb{R}^{2}$ is a planar polygon, then the number of integral points contained in $p \cdot \Delta(p \in \mathbb{N})$ is a polynomial function in $p$ (equal to the Euler characteristic $\chi(X(\Delta), p \bar{C})$ ).

0. Newton-Okounkov bodies. Based on the above exposition, it becomes important to know if there is a bridge allowing to explore any projective variety using convex geometry. Note that the Newton polygon is constructed from a very specific valuation, which associates to a monomial $x^{i} y^{j}$ the vector $(i, j) \in \mathbb{R}^{2}$. Since the picture is local, then it can be said that the Newton polygon $\Delta(f)$ encodes how all the monomials appearing in $f$ vanish along a fixed set of local parameters.

In order to introduce such a convex construction in the global setting one needs to make some changes to the initial construction. First, instead of a local system of parameters one will consider a complete flag on the initial variety. Instead of associating convex sets to functions we will do that for divisors (or line bundles). And lastly and more importantly, due to the fact that we work in the global setting we will need to take the asymptotic version of Newton polygon.

Based on these ideas and inspired by work of Khovanskii from the 1970s, Okounkov [O96] [O00] explained in passing how to associate to an ample divisor a convex set, called the Newton-Okounkov body, encoding how all the sections of all powers of the divisor vanish along a fixed flag. The foundation of this theory was then laid down in whole generality by Lazarsfeld-Mustaţă [LM09] and independently by Kaveh-Khovanskii [KK12].

We introduce the construction of Newton-Okounkov bodies in dimension two for ease of notation, but the higher-dimensional counterpart is done accordingly in an inductive manner.

Let $X$ be a smooth projective surface, and $H$ a Cartier divisor on $X$. Let $(C, x)$ be a flag on $X$, consisting of an irreducible curve $C \subseteq X$ and $x \in C$ a smooth point. We will denote by $\sim$ the linear equivalence of divisors. Then to measure how an effective divisor $D \sim H$ vanishes along the flag $(C, x)$, one constructs a valuation vector $v_{(C, x)}(D)=\left(v_{1}(D), v_{2}(D)\right)$ given by

$$
v_{1}(D)=\operatorname{ord}_{C}(D) \text { and } v_{2}(D)=\operatorname{ord}_{x}\left(\left.\left(D-v_{1}(D) C\right)\right|_{C}\right) .
$$

Finally, the Newton-Okounkov body of this data is defined via

$$
\Delta_{(C, x)}(H) \stackrel{\text { def }}{=} \text { closed convex hull }\left(\bigcup_{m \geqslant 1} \frac{1}{m}\left\{v_{(C, x)}(D) \mid D \sim m H\right\}\right) \subseteq \mathbb{R}^{2} .
$$

If $\vec{v}_{x} \in \mathrm{T}_{x} X$ is a tangency direction at $x$, then one can associate the infinitesimal Newton-Okounkov body $\Delta_{\left(x, \vec{v}_{x}\right)}(H)$ in a similar way. In particular, it can be seen as the Newton-Okounkov body of $H$ defined by a flag on the blow-up of $X$ at point $x$.

Since we work in the global setting and take the asymptotic view, it turns out that these convex sets satisfy very good properties.

Properties. (1) The sets $\Delta_{(C, x)}(H), \Delta_{\left(x, \vec{v}_{x}\right)}(H) \subseteq \mathbb{R}^{2}$ are always compact and closed. On surfaces they turn out to be always polygons, but they can be badly shaped in higher-dimensions. 
(2) $\Delta_{(C, x)}(p H)=p \cdot \Delta_{(C, x)}(H)$ for any integer $p>0$. In particular, one can define the Newton-Okounkov body for a $\mathbb{Q}$-divisor and by continuity for any $\mathbb{R}$-divisor.

(3) We have $H \equiv_{\mathbb{R}} H^{\prime}$ if and only if $\Delta_{(C, x)}(H)=\Delta_{(C, x)}\left(H^{\prime}\right) \forall(C, x)$ on $X$. The first condition also implies that $\Delta_{\left(x, \vec{v}_{x}\right)}(H)=\Delta_{\left(x, \vec{v}_{x}\right)}\left(H^{\prime}\right) \forall\left(x, \vec{v}_{x}\right) \in X \times \mathrm{T}_{x} X$, but not viceversa.

(4) $\int_{\Delta_{(C, x)}(H)} 1 d t d y=\frac{1}{2} \operatorname{vol}_{X}(H)\left(:=\lim _{m \rightarrow \infty} \frac{h^{0}(X, m H)}{m^{2}}\right)$, for any $(C, x)$.

(5) If $f \in \mathbb{C}[x, y]$ is a non-zero polynomial with Newton polygon $\Delta$, then there exists a flag ( $T$-invariant) on $X(\Delta)$ with respect to which the Newton-Okounkov polygon of $\bar{C}$ is a translate of $\Delta$.

1. Convexity properties of asymptotic invariants. One of the first main applications of Newton-Okounkov bodies, pioneered by Okounkov in [O96] and [O00], is that this theory explains well why some asymptotic invariants in algebraic geometry satisfy properties that seem to come from a convex geometric world. This is well explained by the following example.

Example (Hodge index theorem). Let $X$ be a smooth projective surface, $H$ an ample class on $X$ and $C \subseteq X$ an irreducible curve with $C^{2}>0$. Choose $x \in C$ a random smooth point. Then, by Serre vanishing and the definition of Newton-Okounkov polygons, one knows that

$$
O=(0,0), A=(0,(H . C)) \in \Delta_{(C, x)}(H) .
$$

By the same token, the line $l$ passing through $A$ and having slope $\alpha=-\left(C^{2}\right)$ is a supporting line for the polygon $\Delta_{(C, x)}(H)$. Thus by convexity one has the following inclusion

$$
\Delta_{(C, x)}(H) \subseteq \triangle O A B, \text { where } B=\left(\frac{(H \cdot C)}{C^{2}}, 0\right) .
$$

Computing the areas of both polygons this gives the following inequality

$$
\left(C^{2}\right) \cdot\left(H^{2}\right) \leqslant(H . C)^{2},
$$

i.e. Hodge index theorem.

The basic principle, underlined in the example, is to use Newton-Okounkov bodies to deduce Hodge index theorem and related statements as a consequence of Brunn-Minkowski inequality for volumes of convex sets. In his ground-breaking proof of log-concavity property of the coefficients of the chromatic polynomial of a graph, Huh [H12] gives in passing a simple proof of a higher-dimensional version of Hodge index theorem. Using the same idea, one finds in [LM09] and [KK12] a short proof of the log-concavity property of the volume function of divisors. Furthermore, the authors of [LM09] also find an elegant proof of Fujita approximation of the volume of a divisor based solely on semigroup theory and convex geometry.

This theory has also rendeered clear how to prove such results in the arithmetic world, which are much more difficult to tackle than in complex geometry. For example, Chen [C14] uses a probabilistic and convex geometric approach to give a unified proof of a stronger Hodge index inequality both in the algebraic geometry world (over the complex numbers) and the arithmetic geometry one (over algebraic number fields).

2. Local positivity of divisors through convex geometry. In order to obtain other applications of Newton-Okounkov bodies in algebraic geometry one doesn't need to dig that deep. Going back to property 3 above, we know that the collection of all of them for a fixed divisor serves as an universal numerical invariant. 
Since many interesting properties of the divisor, like positivity for example, are numerical in essence, it becomes natural to ask if they can be translated in the language of Newton-Okounkov bodies.

This philosophy is fully carried out by A. Küronya and the author in [KL14], [KL15A] and [KL15B]. The goal was to see how one can translate positivity properties of the divisor on any algebraic variety, such as ampleness and nefness, into the convex geometry realm.

For a better understanding, we introduce some notation. For positive real number $\lambda, \xi>0$ set

$$
\Delta_{\lambda} \stackrel{\text { def }}{=}\left\{(t, y) \in \mathbb{R}_{+}^{2} \mid t+y \leqslant \lambda\right\} \quad \text { and } \Delta_{\xi}^{*} \stackrel{\text { def }}{=}\left\{(t, y) \in \mathbb{R}_{+}^{2} \mid 0 \leqslant t \leqslant \xi, 0 \leqslant y \leqslant t\right\} .
$$
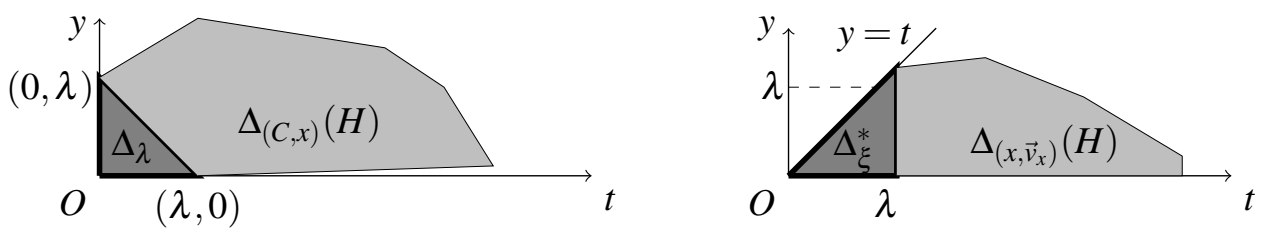

The local and infinitesimal picture of ampleness.

Under these circumstances, the dimensional two case is explained in the following theorem:

Theorem 1. Let $X$ be a smooth projective surface and $H$ an $\mathbb{R}$-divisor on $X$. Then

(i) (Local) $H$ is nef $\Leftrightarrow \forall x \in X$ there exists a flag $(C, x)$ such that $(0,0) \in \Delta_{(C, x)}(H)$.

$H$ is ample $\Leftrightarrow \forall x \in X \exists(C, x)$ and $\lambda>0$ such that $\Delta_{\lambda} \subseteq \Delta_{(C, x)}(H)$.

(ii) (Infinitesimal) $H$ is nef $\Leftrightarrow$ for all $x \in X \exists \vec{v}_{x} \in \mathrm{T}_{x} X$ such that $(0,0) \in \Delta_{\left(x, \vec{v}_{x}\right)}(H)$.

$$
H \text { is ample } \Leftrightarrow \forall x \in X \exists \vec{v}_{x} \in \mathrm{T}_{x} X \text { and } \xi>0 \text { such that } \Delta_{\xi}^{*} \subseteq \Delta_{\left(x, \vec{v}_{x}\right)}(H) \text {. }
$$

Remark 2. The local picture generalizes to any algebraic smooth surface a combinatorial characterization of T-invariant ample/nef divisors on toric surfaces given in terms of classical Newton polygon. Moreover, the condition of ampleness in the local setting can be seen as an intermediate criteria sitting between the classical cohomological version of Serre and the numerical criterion of Nakai-Moishezon-Kleiman (see Chapter 1 of [PAG] for a nice introduction to this classical material). On the other hand, the fact that positivity of divisors can be detected from information given by tangency direction is completely new in the literature.

Remark 3. The infinitesimal picture holds also in higher dimensions, as proved by Küronya and the author in [KL15B]. On the other hand, the local picture seems to be harder to deal with and we attempt in [KL15A] to give a weaker version of Theorem 1.(i).

Since ampleness can be seen through the convex geometry of Newton-Okounkov bodies, then one can easily be lead to the question whether it is possible to read off numerical invariants of the divisor from these convex sets. A classical invariant, measuring how many jets are asymptotically separated by the divisor at a fixed point, is the Seshadri constant. It was introduced by Demailly in the 90s in his work on Fujita conjecture, and is defined to be

$$
\varepsilon(H ; x) \stackrel{\text { def }}{=} \sup _{x \in C \subseteq X} \frac{(H . C)}{\operatorname{mult}_{x}(C)},
$$

where $x \in X$ is a point and $H$ is an ample divisor on $X$. One of the main results of [KL14] ([KL15B] in higher-dimensions) is that the Seshadri constant can be seen on any infinitesimal Newton-Okounkov body defined at the base point $x$. 
Theorem 4. If $H$ is an ample $\mathbb{R}$-divisor on $X$ and $\left(x, \vec{v}_{x}\right) \in X \times \mathrm{T}_{x} X$, then

$$
\varepsilon(H ; x)=\max \left\{\xi>0 \mid \Delta_{\xi}^{*} \subseteq \Delta_{\left(x, \vec{v}_{x}\right)}(H)\right\} .
$$

In particular, the right-hand side does not depend on the tangecy direction.

Remark 5. It is worth noting here that the volume of the divisor $H$ and its largest asymptotic multiplicity can also be seen on a fixed infinitesimal Newton-Okounkov body. This in turn leads to the following natural question: Is it possible to translate questions about one invariant to easier to handle questions of another invariant that can be read off from the same convex set?

In a project with $\mathrm{A}$. Küronya and $\mathrm{C}$. Maclean we tackle this issue. If $H$ is an ample Cartier divisor on a surface $X$ and $x \in X$ is a point, then there exists a three-fold $Y$ and another Cartier divisor $B$ on $Y$ such that

$$
\varepsilon(H ; x) \in \mathbb{Q} \Leftrightarrow \operatorname{vol}_{Y}(B) \in \mathbb{Q} .
$$

Rationality of Seshadri constants is an old, folklore, and still open question. The equivalence reduces this to rationality of the volume of a Cartier divisor. On the other hand, if either the associated ring of the divisor $B$ is finitely generated or there exists a Zariski decomposition, then the volume has to be rational. Thus our initial question can be linked to problems arising in birational geometry, where much has been developed on the problem of finitely generatedness of the canonical ring.

It has long been known that a divisor has better local positivity properties at a very generic choice of the base point oppose to what is happenning at an arbitrary one. For example, an interesting result of Ein-Lazarsfeld from the 90s says that for an ample Cartier divisor $H$, the Seshadri constant $\varepsilon(H ; x) \geqslant 1$ when $x \in X$ is very generic (outside of coutably many curves on $X$ ). If one considers an arbitrary choice of the point, then the Seshadri constant can take on arbitrarily small positive rational values.

So, it becomes natural to ask what can be said about the shape of infinitesimal Newton-Okounkov bodies of an ample Cartier divisor when the base point is taken to be very generic. This has been tackled in [KL14] and can be philosophically explained as follows:

Theorem 6. Let $X$ be a projective surface and $H$ an ample Cartier divisor on $X$. Let $x \in X$ be a very generic point and $\vec{v}_{x} \in \mathrm{T}_{x} X$ a generic tangency direction. Then either we have the inclusion

$$
\Delta_{\left(x, \vec{v}_{x}\right)}(H) \subseteq \triangle O A B,
$$

where $O=(0,0), A=(\varepsilon, \varepsilon)$ and $B=(\mu, 0)$ with $|\mu-\varepsilon| \ll 1$, or there exists a curve $C \subseteq X$ smooth at $x$ with degree $(H, C)$ being small.

Remark 7. Using the Euclidean volumes of the two convex shapes in Theorem 6 one deduces very strong conditions for lower bounds on Seshadri constants at very generic point. For example, Szemberg has conjectured that for a generic surface $X \subseteq \mathbb{P}^{3}$ of degree $N \geqslant 5$ these lower bounds depend on the primitive solution to the Pell's equation $p^{2}-q^{2} N=1$. So, using Theorem 6 one can prove Szemberg conjecture for infinitely many choices of $N$. This and other related problems are tackled in a joint work of the author with A. Küronya and F. Bastianelli.

3. Divisors with nice singularities and convex geometry. In many groundbreaking results in the last thirty years or so, ranging from diophantine approximation to birationcal classification of algebraic varieties, from Kähler to projective geometry, one important step is the ability to find effective divisors that have nice singularities at a given point. In algebraic geometry these ideas have been pioneered by Mori, Kollar, Demailly, Siu and others and is one of the most powerful techniques in modern algebraic geometry. 
On the other hand, going back to the definition of Newton-Okounkov bodies, we know that they encode how all the sections for any power of the divisor vanish along a fixed flag. Thus, one can hope that one might be able to obtain criteria for finding divisors with nice singularities just by looking at these convex sets.

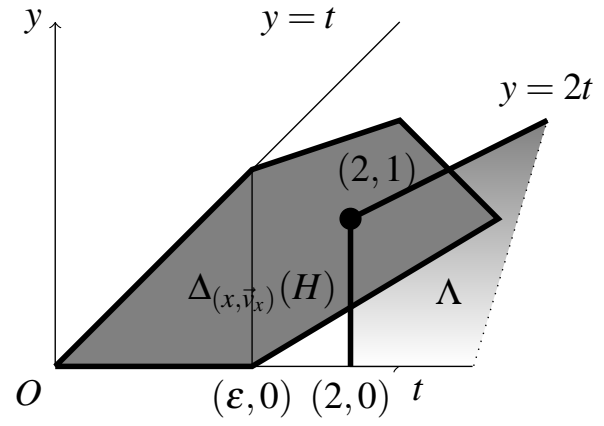

Let $\Lambda \stackrel{\text { def }}{=}\left\{(t, y) \in \mathbb{R}^{2} \mid t \geqslant 2, t \geqslant 2 y \geqslant 0\right\}$. In [KL15C] we give a strong criteria for finding divisors with "nice" singularities in terms of convex geometry of the infinitesimal Newton-Okounkov bodies.

Theorem 8. Let $H$ be an ample $\mathbb{Q}$-divisor on a smooth projective surface $X$. If

$$
\text { interior of }\left(\Delta_{\left(x, \vec{v}_{x}\right)}(H) \bigcap \Lambda\right) \neq \varnothing \text { (as in the figure above), }
$$

for any $\vec{v}_{x} \in \mathrm{T}_{x} X$, then there exists an effective $\mathbb{Q}$-divisor $D \equiv H$ with "nice" singularities at $x$, i.e. the multiplier ideal $\mathscr{J}(X ; D)$ is equal locally (around $x$ ) to the maximal ideal of the point $x$.

Taking into account the statement of Theorem 8, suppose that we are not able to find a divisor with "nice" singularities. Then there exists a tangency direction $\vec{v}_{x} \in \mathrm{T}_{x} X$ for which we know that the Newton-Okounkov polygon $\Delta_{\left(x, \vec{v}_{x}\right)}(H)$ sits above a line passing through the point $(2,1)$. Due to convexity and Theorem 4 , this implies that the Seshadri constant $\varepsilon(H ; x)$ is quite small.

On the other hand, if $H$ is Cartier and $x \in X$ a very generic point, then we can apply Theorem 6. So, either $\varepsilon(H ; x)$ is large or there exists a curve $C \subseteq X$ smooth at $x$ and $(H . C)$ small. This idea was used in [KL15C] to deduce a nice geometric criteria for finding effective divisors with "nice" singularities at very generic points.

Corollary 9. Let $X$ be a smooth surface and $H$ an ample Cartier divisor on $X$ with $\left(L^{2}\right) \geqslant 5(p+2)^{2}(p \in \mathbb{N})$. If $x \in X$ is a very general point and there is no irreducible curve $C \subseteq X$ smooth at $x$ with $1 \leqslant(L \cdot C) \leqslant p+2$, then there exists an effective $\mathbb{Q}$-divisor $D \equiv \frac{1}{p+2} L$ with "nice" singularities as in Theorem 8.

This kind of statements are important, since it translates the condition of existence of divisors with "nice" singularities to a geometric one, the non-existence of curves of small degree. Furthermore, its applications should be manifold. Below we give a surprising application to the study of syzygies on abelian surfaces.

4. Convex geometry and syzygies on abelian surfaces As in the proof of Pythagorean theorem, where one uses convex geometry to explore algebraic equations, we close the circle by explaining how all the ideas above can be used to understand the syzygies on abelian surfaces.

Historically, going back to Hilbert, in order to understand algebraically a subvariety $X \subseteq \mathbb{P}^{n}$ one studies

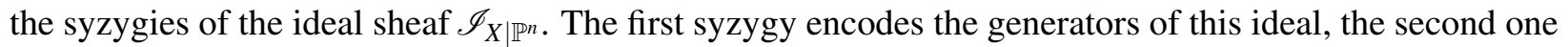
the relationships between these generators etc. Green has introduced a way of how to see the simplest form of syzygies through properties $N_{p}$. For example, $N_{0}$ says that the embedding $X \subseteq \mathbb{P}^{n}$ is projectively normal, $N_{1}$ - the ideal sheaf $\mathscr{I}_{X \mid \mathbb{P}^{n}}$ is generated by quadrics, $N_{2}$ - the relationships between these quadrics are only of linear form etc. (see Chapter 1.8 from [PAG] for a nice introduction in these circle of ideas). 
Using all the ideas explained in this survey, we have found in [KL15C] a very interesting criteria for property $N_{p}$ to be satisfied for abelian surfaces in terms of non-existence of elliptic curves of small degrees. Thus, we have build a bridge between the algebraic and geometric world of an abelian surface, and convex geometry has played a paramount role in this.

Theorem 10. Let $p \geqslant 0$ be a natural number, $X$ a complex abelian surface, and $L$ an ample line bundle on $X$ with $\left(L^{2}\right) \geqslant 5(p+2)^{2}$. Then the following two conditions are equivalent:

(1) $X$ does not contain an elliptic curve $C$ with $\left(C^{2}\right)=0$ and $1 \leqslant(L \cdot C) \leqslant p+2$.

(2) The line bundle L satisfies property $N_{p}$.

Remark 11. It is proved in [LPP11] that on an abelian space property $N_{p}$ is implied by the existence of a divisor $D=\frac{1}{p+2} L$ with just a bit "nicer" singularitiess as those seen in Theorem 8 , i.e. the multiplier ideal $\mathscr{J}(X, D)$ is globally (and not only locally) the maximal ideal of the origin $0 \in X$. Philosophically, Theorem 10 follows by refining the tools used in the proof of Corollary 9.

Acknowledgments. Most of the material presented here is joint work with Alex Küronya. The author would like to thank him for the support, advices and many interesting conversations on the subject. Furthermore, the author would like to thank the organizers of the "Workshop on Positivity and Valuations" in Barcelona for organizing such a beautiful event and for giving the opportunity to talk about the ideas above.

\section{REFERENCES}

[C14] H. Chen, Inegalité d'indice de Hodge en géométrie et arithmétique: une approche probabiliste (2014). $\uparrow(\mathrm{document)}$

[H12] June Huh, Milnor numbers of projective hypersurfaces and the chromatic polynomial of graphs, J. Amer. Math. Soc. 25 (2012), no. 3, 907-927. $\uparrow($ document)

[KK12] Kiumars Kaveh and A. G. Khovanskii, Newton-Okounkov bodies, semigroups of integral points, graded algebras and intersection theory, Ann. of Math. (2) 176 (2012), no. 2, 925-978. $\uparrow$ (document)

[Ko07] János Kollár, Lectures on resolution of singularities, Annals of Mathematics Studies, vol. 166, Princeton University Press, Princeton, NJ, 2007. $\uparrow$ (document)

[KL14] Alex Küronya and Victor Lozovanu, Local positivity of linear series on surfaces. (2014). arXiv:1411.6205. $\uparrow($ document)

[KL15A] Alex Küronya and Victor Lozovanu, Positivity of Line bundles and Newton-Okounkov bodies (2015). arXiv:1506.06525. $\uparrow($ document), 3

[KL15B]_, Infinitesimal Newton-Okounkov bodies and jet separation (2015). arXiv:1507.04339. $\uparrow$ (document), 3

[KL15C] _ _ A Reider-type theorem for higher syzygies on abelian surfaces (2015). arXiv:1509.08621. $\uparrow$ (document)

[PAG] Robert Lazarsfeld, Positivity in algebraic geometry. I-II, Ergebnisse der Mathematik und ihrer Grenzgebiete. 3. Folge., vol. 48-49, Springer-Verlag, Berlin, 2004. $\uparrow 2$, (document)

[LM09] Robert Lazarsfeld and Mircea Mustaţă, Convex bodies associated to linear series, Ann. Sci. Éc. Norm. Supér. (4) 42 (2009), no. 5, 783-835 (English, with English and French summaries). $\uparrow$ (document)

[LPP11] Robert Lazarsfeld, Giuseppe Pareschi, and Mihnea Popa, Local positivity, multiplier ideals, and syzygies of abelian varieties, Algebra Number Theory 5 (2011), no. 2, 185-196. $\uparrow 11$

[O96] Andrei Okounkov, Brunn-Minkowski inequality for multiplicities, Invent. Math. 125 (1996), no. 3, 405-411. $\uparrow$ (document)

[O00] _ Why would multiplicities be log-concave?, The orbit method in geometry and physics (Marseille, 2000), Progr. Math., vol. 213, Birkhäuser Boston, Boston, MA, 2003, pp. 329-347. 个(document)

Victor Lozovanu, Université de CAen Normandie, Laboratoire de Mathématiques “N. OResme”, CAen, FRANCE

E-mail address: victor. lozovanu@gmail.com 